\title{
Mathematical Models and the Noise -Vibration of the Gearboxes
}

\author{
Dr. Cao Hung Phi \\ Vinh Long University of Technology Education \\ 73 Nguyen Hue Street, 2nd Ward, Vinh Long City 91000, Vietnam \\ caohungphi@yahoo.com
}

\begin{abstract}
Noise and vibration from cars is fairly a complex field occurring in a right frequency range: vibration to noise. In practice, there is a variety of reasons causing noise inside a normal sealed gearbox. According to the J.D.Smith's (2) analysis, the primary cause of noise is from backlash gaps between teeth when mashing. The friction effects of gear teeth generate vibration, and are then transmitted to a primary rolling bearing (4). In the gearbox internal responses, all of the manufacturing errors, design errors and tooth and gear deflections, etc...are called Transmission Error (TE). TE will be passing the gearbox whole system to the end rolling bearing of a primary shaft. The gear operation of a modeled gearbox showed in Fig. 4 vibrates with four free levels. Generalized coordinates $\varphi_{1} \ldots \varphi_{5}$ are the gear angular velocity from 1 to 5 respectively indicated in Fig.4. The symbols $\mathrm{J}_{1}\left(\mathrm{~J}_{21}, \mathrm{~J}_{22}\right),\left(\mathrm{J}_{31}, \mathrm{~J}_{32}\right),\left(\mathrm{J}_{41}, \mathrm{~J}_{42}\right), \mathrm{J}_{5}$ are the gear moments from 1 to 5 . Responding to the wheel circle radius symbolized $\mathrm{r}_{1}, \mathrm{r}_{21}, \mathrm{r}_{22} \ldots \mathrm{r}_{5}$ and $\mathrm{I}_{1}, \mathrm{I}_{2}, \mathrm{I}_{3}, \mathrm{I}_{4}$, are vibrating forces. The symbols $\mathrm{c}_{1}, . . \mathrm{c}_{4} ; \mathrm{k}_{1}, \ldots \mathrm{k}_{4}$ are the viscous fluid damper and the mating gear elasticity (Fig.5). Applying the Hezt's collision theory, the momentum principle, the Newton's second law of motion, and the author has developed a vibrating equation of the gearbox transmission of four vibrating levels as the following equation (01)

To survey vibration in a modeled gearbox, ones need to consider the equation (01) and receive $\varphi_{1} \ldots \varphi_{5}$ then deal with $\ddot{x}_{5}=r_{5} \ddot{\varphi}_{5}$ and according to the formula (12) gives the velocity vector in XYZ of primary bearing housing. With constructing mathematical models, ones can recognize vibration in a certain gearbox when changing the gearbox theoretical design and specifications with convenience, which supports design processes more effectively and quickly.
\end{abstract}

Keywords: Gearbox, Noise-Vibration, Force, Transmission
Error (TE), Moment, Equation.

\section{Modeled gearbox}

A modeled gearbox dealing with noise-vibration is the one which is employed for cars less than 3 Tons, and 5 shift levers with a reverse lever and two-speed levels (fast and slow) creating a transmission range in ten shift levers. The shift levers $2 ; 3$ and $4 ; 5$ contain a synchromesh.

Gear ratios:

$$
\begin{gathered}
\bullet \quad \text { Fastness }\left(\mathrm{i}_{\mathrm{n}}=1\right) \\
\begin{array}{c}
\mathrm{I}_{1}=7.31, \mathrm{I}_{2}=4.31, \mathrm{I}_{3}=2.54, \mathrm{I}_{4}=1.54, \mathrm{I}_{5}=1 \\
\bullet \quad \text { Slowness }\left(\mathrm{I}_{\mathrm{c}}=1.56\right)
\end{array} \\
\mathrm{I}_{1}=11.4, \mathrm{I}_{2}=6.72, \mathrm{I}_{3}=3.82, \mathrm{I}_{4}=2.4, \mathrm{I}_{5}=1.56
\end{gathered}
$$

The last transmission ratio of gears is 6.57 .

\subsection{Characteristics}

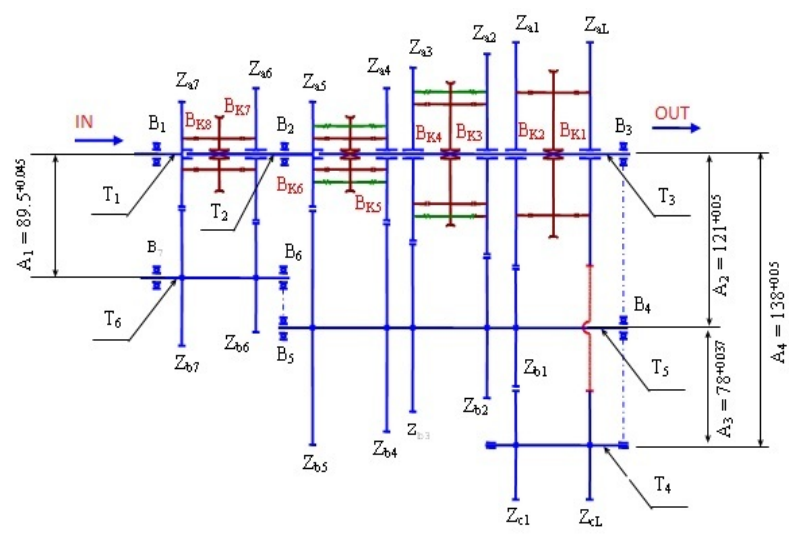

Fig.1. Modeled gears' transmission 


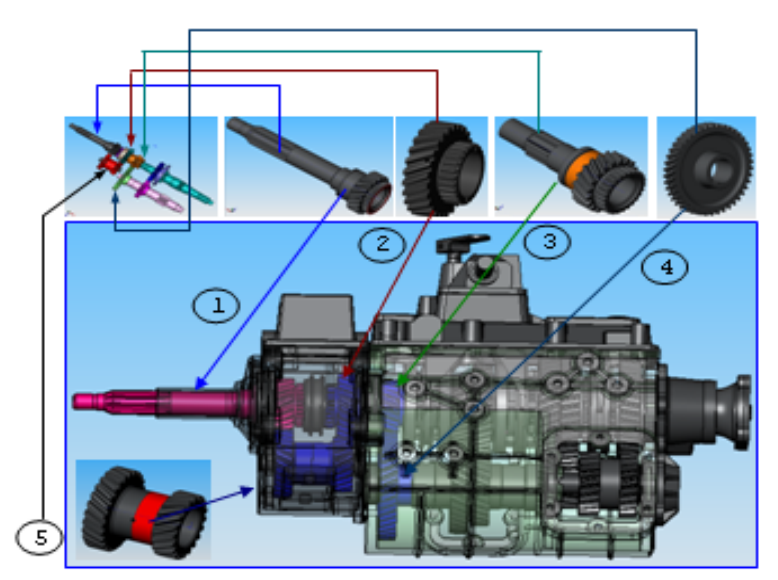

Fig. 2. Modeled gearbox's components

\subsection{Power transmission paths}

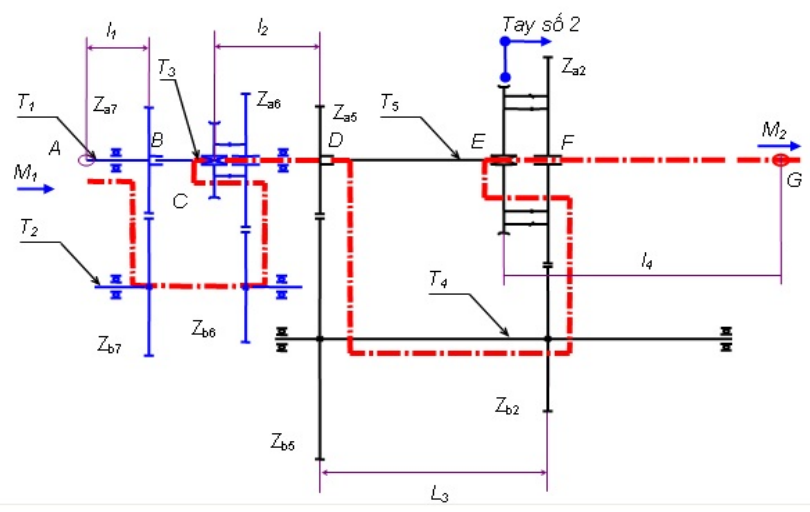

Fig.3. Transmission of a modeled gearbox $\mathrm{N}_{0} 2$

\section{Descriptions:}

+ The modeled transmission comprises 4 successive teeth pairs:

$$
i_{2}=\frac{Z_{a 2}}{Z_{b 2}} \cdot \frac{Z_{b 5}}{Z_{a 5}} \cdot \frac{Z_{a 6}}{Z_{b 6}} \cdot \frac{Z_{b 7}}{Z_{a 7}}=\frac{39}{20} \bullet \frac{42}{19} \bullet \frac{24}{21} \cdot \frac{26}{19}=6.7413
$$

+ The system has 5 shafts: $\mathrm{T}_{1}, \mathrm{~T}_{2}, \mathrm{~T}_{3}, \mathrm{~T}_{4}, \mathrm{~T}_{5}$

+ The input and output moments:

$$
\mathrm{M}_{1} \text { : moment into secondary shafts }
$$

$\mathrm{M}_{2}$ : moment into primary shafts

In the gearbox internal responses, all of the manufacturing errors, design errors and teeth and gear deflections, etc...are called Transmission Error (TE). TE will be passing the gearbox whole system to the end rolling bearing of a primary shaft. The truck gearbox is surveyed with gearcase $\delta>5 \mathrm{~mm}$, components therefore are solid, not cell. Hence, dominant noise sources are transmitted to the gearcase emitting sounds, but these are insignificant [3]. Vibration in the gearbox will be transmitted to a primary bearing housing. The noisiest source is at the position of the primary bearing housing (Fig.6). J.Derek Smith indicated that this is the noisiest and highest vibrating position (2). The measures at gearbox various positions showed that the position of a primary bearing housing results in the noisiest and highest vibrating.

\section{Form a linear equation system of the gearbox vibration}

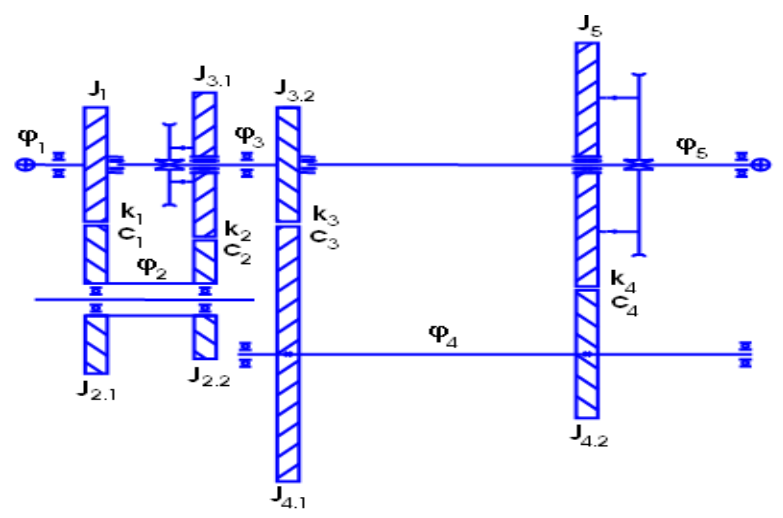

Fig.4. Modeled transmission of a gearbox

\subsection{Forming linear equations of the gearbox vibration:}

The transmission between gears showed in Fig.4 is a system with the four freely vibrating levels. To develop linear equations of the gearbox vibrating, ones theorize:

The gear viscous fluids and materials on teeth surfaces work with linear principles. The interactions between meshing teeth can therefore be described in the model (Fig.5). The shafts are solidly considered.

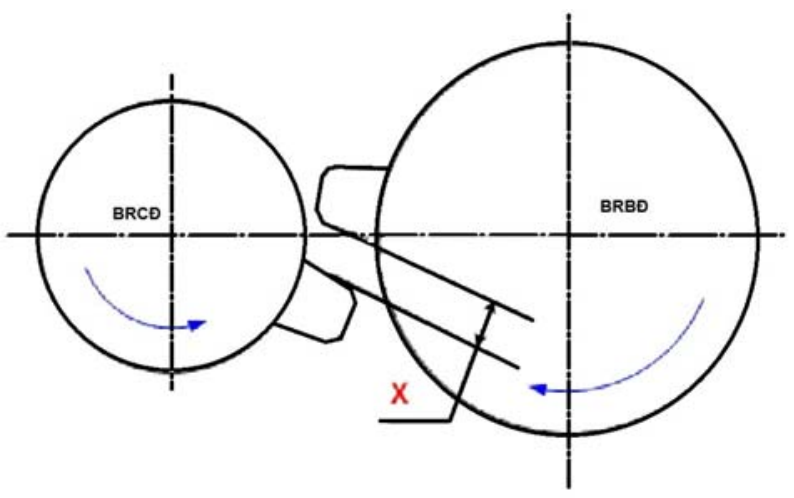

Fig.5. Model of meshing teeth

Generalized coordinates $\varphi_{1} \ldots \varphi_{5}$ are the gear angular velocity from 1 to 5 respectively indicated in Fig.4. The symbols $\mathrm{J}_{1}\left(\mathrm{~J}_{21}, \mathrm{~J}_{22}\right),\left(\mathrm{J}_{31}, \mathrm{~J}_{32}\right),\left(\mathrm{J}_{41}, \mathrm{~J}_{42}\right), \mathrm{J}_{5}$ are the gear moments from 1 to 5 . Responding to the wheel circle radius 
symbolized $r_{1}, r_{21}, r_{22} \ldots r_{5}$ and $I_{1}, I_{2}, I_{3}, I_{4}$, are vibrating forces. The symbols $c_{1}, . . c_{4} ; k_{1}, \ldots k_{4}$ are the viscous fluid damper and the mating gear elasticity (Fig.4).

Analyzing teeth mashing processes, each pair of teeth respectively mates while they are in contact. The processes take place with a certain circle " $\mathrm{T}$ " as gears reel called a $\mathrm{T}$ circle process.

From the modeled gearbox, the author has analyzed the vibration forces between the mashing teeth when backlash gaps occur in angular movements, and the author has then developed a mathematical model using momentum principles to produce differential equations of gearbox transmission and vibrations with the 4 following levels:

$$
\begin{aligned}
& j_{1} \ddot{\varphi}_{1}+r_{1}\left[c_{1}\left(r_{1} \dot{\varphi}_{1}-r_{21} \dot{\varphi}_{2}\right)+k_{1}\left(r_{1} \varphi_{1}-r_{21} \varphi_{2}\right)\right]=M_{1}+r_{1} I_{1} \\
& \left(j_{21}+j_{22}\right) \ddot{\varphi}_{2}-r_{21}\left[c_{1}\left(r_{1} \dot{\varphi}_{1}-r_{21} \dot{\varphi}_{2}\right)+k_{1}\left(r_{1} \varphi_{1}-r_{21} \varphi_{2}\right)\right] \\
& +r_{22}\left[c_{2}\left(r_{22} \dot{\varphi}_{2}-r_{31} \dot{\varphi}_{3}\right)+k_{2}\left(r_{22} \varphi_{2}-r_{31} \varphi_{3}\right)\right]=r_{21} I_{1}+r_{22} I_{2} \\
& \left(j_{31}+j_{32}\right) \ddot{\varphi}_{3}-r_{31}\left[c_{2}\left(r_{22} \dot{\varphi}_{2}-r_{31} \dot{\varphi}_{3}\right)+k_{2}\left(r_{22} \varphi_{2}-r_{31} \varphi_{3}\right)\right] \\
& +r_{32}\left[c_{3}\left(r_{32} \dot{\varphi}_{3}-r_{41} \dot{\varphi}_{4}\right)+k_{3}\left(r_{32} \varphi_{3}-r_{41} \varphi_{4}\right)\right]=r_{31} I_{2}+r_{32} I_{3} \\
& \left(j_{41}+j_{42}\right) \ddot{\varphi}_{4}-r_{41}\left[c_{3}\left(r_{32} \dot{\varphi}_{3}-r_{41} \dot{\varphi}_{4}\right)+k_{3}\left(r_{32} \varphi_{3}-r_{41} \varphi_{4}\right)\right] \\
& +r_{42}\left[c_{4}\left(r_{42} \dot{\varphi}_{4}-r_{5} \dot{\varphi}_{5}\right)+k_{4}\left(r_{42} \varphi_{4}-r_{5} \varphi_{5}\right)\right]=r_{41} I_{3}+r_{42} I_{4} \\
& j_{5} \ddot{\varphi}_{5}+r_{5}\left[c_{4}\left(-r_{42} \dot{\varphi}_{4}+r_{5} \dot{\varphi}_{5}\right)+k_{4}\left(-r_{42} \varphi_{4}+r_{5} \varphi_{5}\right)\right] \\
& =M_{2}+r_{5} I_{4}
\end{aligned}
$$

\section{If ones symbolize:}

$\mathrm{J}_{1}=\mathrm{j}_{1} ; \mathrm{J}_{2}=\mathrm{j}_{21}+\mathrm{j}_{22} ; \mathrm{J}_{3}=\mathrm{j}_{31}+\mathrm{j}_{32} ; \mathrm{J}_{4}=\mathrm{j}_{41}+\mathrm{j}_{42} ; \mathrm{J}_{5}=\mathrm{j}_{5} ;$

$\mathrm{x}_{1}=\mathrm{r}_{1} \varphi_{1}-\mathrm{r}_{21} \varphi_{2} ; \mathrm{x}_{2}=\mathrm{r}_{22} \varphi_{2}-\mathrm{r}_{31} \varphi_{3} ; \quad \mathrm{x}_{3}=\mathrm{r}_{32} \varphi_{3}-\mathrm{r}_{41} \varphi_{4}$;

$x_{4}=r_{42} \varphi_{4}-r_{5} \varphi_{5} ; \quad x_{5}=r_{5} \varphi_{5}$

$\mathrm{X}$ is relative movements between a pair of teeth

Deduct:

$$
\begin{aligned}
& r_{5} \varphi_{5}=x_{5} \\
& r_{42} \varphi_{4}=x_{4}+x_{5} \\
& r_{32} \varphi_{3}=x_{3}+r_{41} \varphi_{4}=x_{3}+\frac{r_{41}}{r_{42}}\left(x_{4}+x_{5}\right) \\
& r_{22} \varphi_{2}=x_{2}+r_{31} \varphi_{3}=x_{2}+\frac{r_{31}}{r_{32}}\left[x_{3}+\frac{r_{41}}{r_{42}}\left(x_{4}+x_{5}\right)\right] \\
& r_{1} \varphi_{1}=x_{1}+r_{21} \varphi_{2}=x_{1}+\frac{r_{21}}{r_{22}}\left\{x_{2}+\frac{r_{31}}{r_{32}}\left[x_{3}+\frac{r_{41}}{r_{42}}\left(x_{4}+x_{5}\right)\right]\right\}
\end{aligned}
$$


Ones would have linear equations (7):

$$
\begin{aligned}
& \ddot{x}_{5}=\frac{r_{5}^{2}}{J_{5}}\left(c_{4} \dot{x}_{4}+k_{4} x_{4}\right)+\frac{r_{5}}{J_{5}} M_{2}+\frac{r_{5}^{2}}{J_{5}} I_{4} \\
& \ddot{x}_{4}=\frac{r_{41} r_{42}}{J_{4}}\left(c_{3} \dot{x}_{3}+k_{3} x_{3}\right)-\left(\frac{r_{5}^{2}}{J_{5}}+\frac{r_{42}^{2}}{J_{4}}\right)\left(c_{4} \dot{x}_{4}+k_{4} x_{4}\right) \\
& -\frac{r_{5}}{J_{5}} M_{2}+\frac{r_{41} r_{42}}{J_{4}} I_{3}-\left(\frac{r_{5}^{2}}{J_{5}}-\frac{r_{42}^{2}}{J_{4}}\right) I_{4} \\
& \ddot{x}_{3}=\frac{r_{31} r_{32}}{J_{3}}\left(c_{2} \dot{x}_{2}+k_{2} x_{2}\right)-\left(\frac{r_{41}^{2}}{J_{4}}+\frac{r_{32}^{2}}{J_{3}}\right)\left(c_{3} \dot{x}_{3}+k_{3} x_{3}\right) \\
& +\frac{r_{41} r_{42}}{J_{4}}\left(c_{4} \dot{x}_{4}+k_{4} x_{4}\right)+\frac{r_{31} r_{32}}{J_{3}} I_{2}-\left(\frac{r_{41}^{2}}{J_{4}}-\frac{r_{32}^{2}}{J_{3}}\right) I_{3}-\frac{r_{41} r_{42}}{J_{4}} I_{4} \\
& \ddot{x}_{2}=\frac{r_{21} r_{22}}{J_{2}}\left(c_{1} \dot{x}_{1}+k_{1} x_{1}\right)-\left(\frac{r_{31}^{2}}{J_{3}}+\frac{r_{22}^{2}}{J_{2}}\right)\left(c_{2} \dot{x}_{2}+k_{2} x_{2}\right) \\
& +\frac{r_{31} r_{32}}{J_{3}}\left(c_{3} \dot{x}_{3}+k_{3} x_{3}\right)+\frac{r_{21} r_{22}}{J_{2}} I_{1}-\left(\frac{r_{31}^{2}}{J_{3}}-\frac{r_{22}^{2}}{J_{2}}\right) I_{2}-\frac{r_{31} r_{32}}{J_{3}} I_{3} \\
& \left.+\frac{r_{1}}{J_{1}} M_{1}-\left(\frac{r_{21}^{2}}{J_{2}}-\frac{r_{1}^{2}}{J_{1}}\right) I_{1}-\frac{r_{21}^{2}}{J_{2}}+\frac{r_{1}^{2}}{J_{1}}\right)\left(c_{1} \dot{x}_{1}+k_{1} x_{1}\right)+\frac{r_{21} r_{22}}{J_{2}}\left(c_{2} \dot{x}_{2}+k_{2} x_{2}\right)
\end{aligned}
$$

\subsection{Linear equations can be performed as matrices}

$\{\ddot{x}\}+[c]\{\dot{x}\}+[k]\{x\}=\{M\}+[\alpha]\{I\}$

With:

$$
\begin{aligned}
& \{\ddot{x}\}=\left\{\ddot{x}_{1} ; \ddot{x}_{2} ; \ddot{x}_{3} ; \ddot{x}_{4}\right\} ;\{\dot{x}\}=\left\{\dot{x}_{1} ; \dot{x}_{2} ; \dot{x}_{3} ; \dot{x}_{4}\right\} \\
& \{x\}=\left\{x_{1} ; x_{2} ; x_{3} ; x_{4}\right\} ;\{I\}=\left\{I_{1} ; I_{2} ; I_{3} ; I_{4}\right\} ; \\
& \{M\}=\left\{\frac{r_{1}}{J_{1}} M_{1} ; 0 ; 0 ;-\frac{r_{5}}{J_{5}} M_{2}\right\}
\end{aligned}
$$

(c) isthe fourth level square matrix showing vibration reducing coefficients

$$
[c]=\left[\begin{array}{cccc}
c_{1}\left(\frac{r_{21}^{2}}{J_{2}}+\frac{r_{1}^{2}}{J_{1}}\right) & -c_{2} \frac{r_{21} r_{22}}{J_{2}} & 0 & 0 \\
-c_{1} \frac{r_{21} r_{22}}{J_{2}} & c_{2}\left(\frac{r_{31}^{2}}{J_{3}}+\frac{r_{22}^{2}}{J_{2}}\right) & -c_{3} \frac{r_{31} r_{32}}{J_{3}} & 0 \\
0 & -c_{2} \frac{r_{31} r_{32}}{J_{3}} & c_{3}\left(\frac{r_{41}^{2}}{J_{4}}+\frac{r_{32}^{2}}{J_{3}}\right) & -c_{4} \frac{r_{41} r_{42}}{J_{4}} \\
0 & 0 & -c_{3} \frac{r_{41} r_{42}}{J_{4}} & c_{4}\left(\frac{r_{5}^{2}}{J_{5}}+\frac{r_{42}^{2}}{J_{4}}\right)
\end{array}\right]
$$

(k) is the fourth level square matrix being solid matrices

$$
[k]=\left[\begin{array}{cccc}
k_{1}\left(\frac{r_{21}^{2}}{J_{2}}+\frac{r_{1}^{2}}{J_{1}}\right) & -k_{2} \frac{r_{21} r_{22}}{J_{2}} & 0 & 0 \\
-k_{1} \frac{r_{21} r_{22}}{J_{2}} & k_{2}\left(\frac{r_{31}^{2}}{J_{3}}+\frac{r_{22}^{2}}{J_{2}}\right) & -k_{3} \frac{r_{31} r_{32}}{J_{3}} & 0 \\
0 & -k_{2} \frac{r_{31} r_{32}}{J_{3}} & k_{3}\left(\frac{r_{41}^{2}}{J_{4}}+\frac{r_{32}^{2}}{J_{3}}\right) & -k_{4} \frac{r_{41} r_{42}}{J_{4}} \\
0 & 0 & -k_{3} \frac{r_{41} r_{42}}{J_{4}} & k_{4}\left(\frac{r_{5}^{2}}{J_{5}}+\frac{r_{42}^{2}}{J_{4}}\right)
\end{array}\right]
$$

$(\alpha)$ is the fourth level square matrix being vibrating forces

$$
[\alpha]=\left[\begin{array}{cccc}
\frac{r_{21}^{2}}{J_{2}}-\frac{r_{1}^{2}}{J_{1}} & \frac{r_{21} r_{22}}{J_{2}} & 0 & 0 \\
-\frac{r_{21} r_{22}}{J_{2}} & \frac{r_{31}^{2}}{J_{3}}-\frac{r_{22}^{2}}{J_{2}} & \frac{r_{31} r_{32}}{J_{3}} & 0 \\
0 & -\frac{r_{31} r_{32}}{J_{3}} & \frac{r_{41}^{2}}{J_{4}}-\frac{r_{32}^{2}}{J_{3}} & \frac{r_{41} r_{42}}{J_{4}} \\
0 & 0 & -\frac{r_{41} r_{42}}{J_{4}} & \frac{r_{5}^{2}}{J_{5}}-\frac{r_{42}^{2}}{J_{4}}
\end{array}\right]
$$

\section{Analyze linear equations of the gearbox vibration}

Ones can therefore form linear equations from (8) to (11) describing vibration throughout the modeled gearbox. Due to gears inside sealed gearboxes, ones can only examine the first shaft (secondary) and the fifth shaft (primary). The primary shaft vibration is closely connected to bearing vibration. If ones symbolize $\vec{x}_{5}$ being an acceleration vector in the fourth mating pair, the vector result will be $\ddot{x}_{5}=r_{5} \ddot{\varphi}_{5}$. This acceleration is totally transmitted to a primary shaft bearing; vibrating signs can therefore be measured in a primary shaft.

If ones symbolize $\alpha$ being teeth mashing angles $\left(\alpha=20^{\circ}\right)$ and $\beta$ being teeth tilt angles $\left(\beta=34^{0} 13\right.$ ' 12 '), acceleration components can be measured in 3 coordinate systems respectively:

$$
\begin{aligned}
& \ddot{x}_{5 x}=\ddot{x}_{5} \sin \beta \\
& \ddot{x}_{5 y}=\ddot{x}_{5} \cos \beta \sin \alpha \\
& \ddot{x}_{5 z}=\ddot{x}_{5} \cos \beta \cos \alpha
\end{aligned}
$$

Acceleration components (12) are the vibrating signs measured in a primary shaft bearing. 


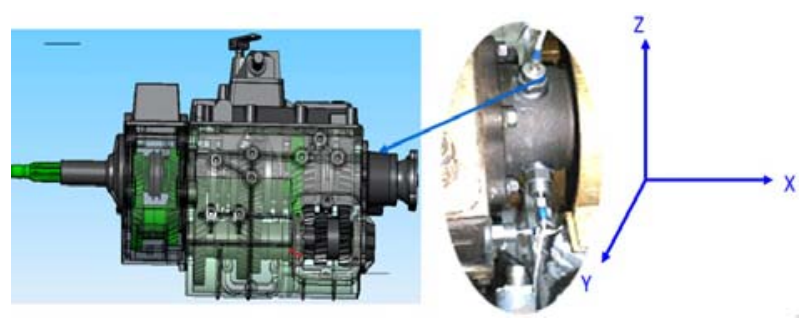

Fig.6.Sensor position measuring noise-vibration.

To survey noise-vibration in a modeled gearbox, ones need to deal with linear equations (1) to receive $\varphi_{1}, \ldots \varphi_{5}$, then $\ddot{x}_{5}=r_{5} \ddot{\varphi}_{5}$, finally an acceleration. Ones can also handle linear equations (6) to have $\left(\mathrm{x}_{1}, . . \mathrm{x}_{4}\right)$, the $\mathrm{x}_{5}$ with the following formula:

$$
\ddot{x}_{5}=\frac{r_{5}^{2}}{J_{5}}\left[M_{2}+r_{5}\left(I_{4}+c_{4} \dot{x}_{4}+k_{4} x_{4}\right)\right]
$$

With the above methods, ones can recognize results about noise-vibration of the gearboxes when changing design, and specifications with convenience in theory, which support the gearboxes design processes more effectively and quickly.

\section{Conclusions}

Noise-vibration in cars appear in the gearbox operating processes, the unsuccessfully mashing gears will generate vibration, which is the cause of backlash gaps, and vibrating processes happen in such a short time called force. Vibrating force excites teeth mating processes causing vibration in a gearbox.

With the hypothesis: the viscous fluids as well as materials on the teeth surfaces work with linear principles. Analyzing the mashing processes of a pair of teeth (Fig.5), applying the function Fourier, the Hezt's collision theory, the Newton's second law of motion, the author has developed the mathematical model describing gearboxes vibration under the differential equations (01).

With this method dealing with linear equations, the author can have $\ddot{\varphi}_{5}$. Ones can therefore recognize vibration in a certain gearbox when changing the gearbox theoretical design and specifications with convenience, which supports design processes more effectively and quickly.

\section{Acknowledgment}

While presenting the issues surrounding Noise-Vibration, mistakes cannot be avoided. Thus, the author apologizes for any omissions and will be pleased to have any advice and comments from others through the email address: caohungphi@yahoo.com; phone: (+84)913612652.

\section{References}

(1) Alec Stocks: "Manual Gearbox Design", 1992/SAE

(2) J. Derek Smith: "Gear Noise and Vibration. Second Edition, Revised and Expanded", Cambridge University, Cambridge, England. Copyright (C) 2003 by Marcel Dekker, Inc. All Rights Reserved.

(3) "Digital sound level meter. Model 8928 obtainable", A.T.P., Tournament Way, Ashby-de-la Zouche, Leics. LE65 2UU, UK002E

(4) M.J. Roberts: "Signal and Systems", McGraw-Hill 2003. 